\title{
Application of a Crossover Equation of State to Describe Phase Equilibrium and Critical Properties of n-Alkanes and Methane/n-Alkane Mixtures
}

P. C. M. Vinhal, Andre; Yan, Wei; Kontogeorgis, Georgios M.

Published in:

Journal of Chemical and Engineering Data

Link to article, DOI:

10.1021/acs.jced.7b00779

Publication date:

2018

Document Version

Peer reviewed version

Link back to DTU Orbit

Citation (APA):

P. C. M. Vinhal, A., Yan, W., \& Kontogeorgis, G. M. (2018). Application of a Crossover Equation of State to Describe Phase Equilibrium and Critical Properties of n-Alkanes and Methane/n-Alkane Mixtures. Journal of Chemical and Engineering Data, 63, 981-993. https://doi.org/10.1021/acs.jced.7b00779

\section{General rights}

Copyright and moral rights for the publications made accessible in the public portal are retained by the authors and/or other copyright owners and it is a condition of accessing publications that users recognise and abide by the legal requirements associated with these rights.

- Users may download and print one copy of any publication from the public portal for the purpose of private study or research.

- You may not further distribute the material or use it for any profit-making activity or commercial gain

- You may freely distribute the URL identifying the publication in the public portal 


\section{Introduction}

Due to the growing demand for energy production, the interest in processes using near-critical and supercritical fluids has increased in the last decades. Near-critical and supercritical fluids are substances for which temperature and pressure conditions are close to or above the critical value and possess special characteristics; i.e. gas-like viscosity and diffusivity, as well as liquid-like density and solvating properties [1]. These properties make them excellent solvents for many applications, e.g. the production of gasoline [2], liquefied petroleum gas (LPG) [3] and methanol [4] from syngas, the production of biofuel using alcohols [5] [6], and the use of supercritical $\mathrm{CO}_{2}$ to improve the recovery factor of oil and gas condensate reservoirs [7] [8].

In the vicinity of a critical point, fluids have an asymptotic singular behavior [9] [10], which is different from the one implied by classical equations of state $(E o S)$. This contrast is due to the long-range fluctuations of the order parameter associated with the critical phase transition, which in vapor-liquid transitions are related to the density of the system [11]. Since the classical models do not take into account the density fluctuations that arise close to the critical point, they are unable to correctly describe the singular behavior of the thermodynamic properties in terms of scaling laws with universal critical exponents and universal scaling functions [12].
Although the limitations of the classical equations of state in the critical region are known for decades [9] [13], these models are still widely applied in the Oil and gas industry [14], due to their simplicity and capacity to accurately describe mixtures of hydrocarbons in subcritical conditions [15]. However, for near/supercritical conditions, modifications to the classical models are necessary to improve the representation of the thermodynamic properties, like the modification of the Benedict-Webb-Rubin (BWR) model by Soave [16], which allowed the correct description of the critical isotherm of pure and binary mixtures of alkanes, and the equation created by Groupe Europeen de Recherchers Gazieres $(G E R G)$ [17] [18]. The GERG models were developed for systems of natural gases and their mixtures, while Soave- $B W R$ can be potentially applied in natural gas and oil systems. Nevertheless, both models are not optimal for systems containing species that possess a highly non-ideal behavior and, as any other analytical EoS, they do not take into account the longrange fluctuations in the order parameter [19]. In fact, more versatile models can be obtained by using the crossover theory [20], which takes into account the effects of the density fluctuations and bridges the gap between non-classical and critical behavior asymptotically close to the critical point and classical behavior far away from the critical region. 
There are two main approaches for describing fluids in a wide range of conditions, namely far from and close to the critical point, based on the renormalization group theory [21].

The first approach was developed by Chen et. al. [22], Kiselev et al. [23] and Jin et al. [24], which incorporates the scaling laws asymptotically close to the critical point and transforms into the regular classical expansion far away from the critical point. Later, Kiselev and coworkers modified his formulation and applied it to several EoS for pure fluids and fluid mixtures [9] [10] [19] [25], including equations for associating compounds [26]. The drawback of this technique is that it uses 3 to 4 additional compound-specific parameters and the crossover schemes are continuously modified and usually applied to a relatively small ensemble of compounds having a similar character, which makes its application for a broader group of species rather difficult [27].

The second method was derived by White and coworkers [13] [20] [28]. They created a recursive procedure based on Wilson's [21] [29] phase-space cell approximation method, using a modified free energy function for the fluid, which extended the range of the original renormalization group theory. This approach introduces fewer parameters, usually 1 to 2 , but the mathematical procedure is computationally demanding. Other authors have applied White's recursive procedure to different equations of state, including a formulation using the Soave-Redlich-Kwong EoS (SRK) [30] [31], the Cubic-Plus-Association EoS (CPA) [32] [33] and different versions of the Statistical Associating Fluid Theory (SAFT) EoS [34] [35] [36].

Since equations of state are extensively applied for modeling the processes in different industries, it is important to have a thermodynamic model capable of describing the properties of the fluids as precisely as possible. In the Oil and Gas industry, the SRK EOS is largely employed to represent the properties of hydrocarbon mixtures. Nevertheless, as discussed before, this classical model is not capable of modeling the thermodynamic behavior of fluids close to the critical point, therefore, for a better representation of processes in regions both far from and close to the critical point, a crossover model should be applied.

In this work, we have utilized the procedure developed by White and coworkers [13] [20] [28] [37] [38] to the $S R K E o S$, in order to correct the representation of the model in the critical region. Furthermore, we have compared the crossover SRK to two types of classical formulation, using the experimental critical parameters and the parameters obtained by fitting experimental data from the saturated vapor-liquid coexistence region, in order to evaluate the advantages of applying density fluctuations into the traditional model.

\section{Thermodynamic model}

White and coworkers developed a mathematical procedure in the form of recursive relations to correct the repulsive Helmholtz energy density of a fluid with a term that takes into account the fluctuations in density that arise near the critical point. Hence, with the use of the recursion relations, the thermodynamic properties approach the singular behavior in the near-critical region, while maintaining the classical behavior far from the critical point [34].

In order to introduce the density inhomogeneities into an equation of state, Prausnitz and co-workers [30] [39] [40] implemented White's procedure by transforming the grand canonical partition function into a functional integral, and then separated the interaction potential into a repulsive and an attractive one:

$$
u(r)=u_{r e p}(r)+u_{a t t}(r)
$$

where $r$ is the molecular distance, $u(r)$ is the total molecular potential, and the subscripts rep and att represent the repulsive and attractive contributions, respectively. The renormalization group $(R G)$ method is applied to the attractive part only since the authors considered that repulsive term contributes entirely to the short wavelength fluctuations. The attractive term is assumed to be composed of short and long wavelength contributions, and the long-range interaction below a certain cutoff length $(L)$ can be precisely described by a mean-field theory.

The effect of short wavelength contributions are calculated by the functional:

$$
F_{s}(\rho)=\int f^{s}(\rho) d \boldsymbol{r}
$$

where $f^{s}$ is the Helmholtz energy density for a homogeneous system with the molar density equals to $\rho$. The functional $F^{s}$ contains the contributions of all fluctuations with short wavelengths [41] and can be represented by any mean-field EoS [34]. The choice of the model is of special importance since the $R G$ procedure does not affect the predictions far from the critical point, hence the underlying model used should be precise for the fluids of interest far from the critical region [35].

Additionally, as $f^{s}$ should only include shortwavelength density fluctuations, it is necessary to take away the other contributions by adding the term $\alpha \rho^{2}$, which results in the expression:

$$
f^{s}=f^{E o S}+\alpha \rho^{2}
$$


where $f^{E o S}$ is the Helmholtz energy per unit volume calculated with the classical $E o S$ and $\alpha$ is the interaction volume, given by:

$$
\alpha=-\frac{1}{2} \int u_{a t t} d \boldsymbol{r}
$$

where $u_{\text {att }}$ is the attractive part of the pair potential.

Far from the critical point, $\alpha$ can be approximated by an analytical function, e.g. the attractive term of the van der Waals EoS. In such regions, the free energy density of the system is correctly represented by a mean-field model, which is the repulsive term $\left(f^{s}\right)$ and the attractive term $\left(\alpha \rho^{2}\right)$, as shown in Equation 3. However, as the critical point is approached, an analytical description of $\alpha$ is no longer possible, requiring a numerical solution [13]. The recursive procedure developed by White and coworkers is applied in $f^{s}$ and the total free energy density is obtained with the inclusion of the attractive term, as given by the equation:

$$
f=f_{n}-\alpha \rho^{2}
$$

where $f_{n}$ is the free energy density incorporating the density fluctuations on all wavelength scales.

The contributions from density fluctuations are taken into account using the phase-space cell approximation [21] [29], in which the free energy density is calculated through a recursive procedure given by:

$$
f_{n}(\rho)=f_{n-1}(\rho)+\delta f_{n}(\rho)
$$

where $f$ is the free is the free energy density and the subscripts are the iteration index. The term which includes the long-wavelength fluctuations, $\delta f_{n}$, is calculated by:

$$
\delta f_{n}(\rho)=-K_{n} \ln \left(\frac{\Omega_{n}^{S}(\rho)}{\Omega_{n}^{l}(\rho)}\right)
$$

where $\Omega_{n}^{S}$ and $\Omega_{n}^{l}$ describe the contributions of the density fluctuations for the short and long range interactions, respectively. The coefficient $K_{n}$ is determined by:

$$
K_{n}=\frac{k_{b} T}{\left(2^{n} L\right)^{3}}
$$

where $k_{b}$ is the Boltzmann constant, $T$ is temperature and $L$ is the cutoff length, a fitted parameter that determines what wavelength size can be accurately evaluated by a mean-field theory.
The terms to account for the contribution of the short and long-range density fluctuations, i.e. $\Omega_{n}^{s}$ and $\Omega_{n}^{l}$, are defined by the expressions:

$$
\begin{gathered}
\Omega_{n}^{S}(\rho)=\int_{0}^{\min \left(\rho, \rho_{\max }-\rho\right)} \exp \left(\frac{-G_{n}^{S}(\rho, x)}{K_{n}}\right) d x \\
\Omega_{n}^{l}(\rho)=\int_{0}^{\min \left(\rho, \rho_{\max }-\rho\right)} \exp \left(\frac{-G_{n}^{l}(\rho, x)}{K_{n}}\right) d x
\end{gathered}
$$

where $G_{n}^{s}$ and $G_{n}^{l}$ are functions that depend on the modified Helmholtz free energy $\left(\bar{f}_{n}^{s}\right.$ and $\left.\bar{f}_{n}^{l}\right)$ are given by:

$$
\begin{aligned}
G_{n}^{s}(\rho) & =\frac{\bar{f}_{n}^{s}(\rho+x)+\bar{f}_{n}^{s}(\rho-x)-2 \bar{f}_{n}^{s}(\rho)}{2} \\
G_{n}^{l}(\rho) & =\frac{\bar{f}_{n}^{l}(\rho+x)+\bar{f}_{n}^{l}(\rho-x)-2 \bar{f}_{n}^{l}(\rho)}{2}
\end{aligned}
$$

Finally, the modified Helmholtz free energy terms are determined by:

$$
\begin{aligned}
& \bar{f}_{n}^{s}=f_{n-1}+\frac{\alpha \rho^{2}}{2^{2 n}}\left(\frac{\psi w^{2}}{2 L^{2}}\right) \\
& \bar{f}_{n}^{l}=f_{n-1}+\alpha \rho^{2}
\end{aligned}
$$

where $\psi$ is an adjustable parameter related to the initial shortest wavelength of the density fluctuations and $w$ refers to the range of the attractive potential. Equations 8 to 14 allow the computation of the correction of the free energy density taking into account the long wavelength density fluctuations.

The first step of the recursive procedure requires the determination of the zero-order solution of the free energy density $\left(f_{0}\right)$, given by Equation 3 . This means that the method starts with a model including only shortwavelength density fluctuations, which is the repulsive term of the free energy $\left(f^{s}\right)$. Hence, the calculation of $f_{0}$ requires the computation of the interaction volume $(\alpha)$. The range of the attractive potential $(w)$ is also needed for the calculation of the modified free energy, Equation 13. These terms can be obtained from a function that represents the attractive intermolecular potential. However, if one uses a phenomenological EoS, e.g. SRK, then it is not possible to assume which part of the model corresponds to the "true" repulsive and attractive terms [30]. This means that in the case of the SRK EoS, in which the pressure explicit equation is:

$$
P=\frac{R T}{1-b \rho}-\frac{a \rho^{2}}{1+b \rho}
$$

the first term of the right-hand side of expression 15 is not only accounting for the repulsive interactions, while the second term is not exclusively representing the attractive interactions. In Equation 15, $P$ is pressure, $R$ is the universal gas constant, $T$ is temperature, $\rho$ is the 
molar density which corresponds to the inverse molar volume $\left(v^{-1}\right)$ and $a$ and $b$ are pure component constants calculated by:

$$
\begin{aligned}
& a=0.42748 \frac{R^{2} T_{c}{ }^{2}}{P_{c}} a(T) \\
& a(T)=\left\{1+\kappa\left[1-\left(\frac{T}{T_{c}}\right)^{0.5}\right]\right\}^{2} \\
& \kappa=0.480+1.57 \omega-0.176 \omega^{2} \\
& b=0.08664 \frac{R T_{c}}{P_{c}}
\end{aligned}
$$

where $T_{c}$ and $P_{c}$ are the critical temperature and pressure of the pure component, respectively, and $\omega$ is the acentric factor.

In order to obtain the correct terms that correspond to the repulsive and attractive interactions from the $S R K$ $E o S$, we compared the cubic model the to the analytic expression for the hard spheres, namely the CarnahanStarling $E o S[42]$ :

$$
Z=\frac{1+\eta+\eta^{2}-\eta^{3}}{(1-\eta)^{3}}
$$

where $Z$ is the compressibility factor and $\eta$, the packing fraction, is equal to $\frac{b \rho}{4}$. The comparison of the two models is done by subtracting the attractive part of the free energy, i.e. $-\alpha \rho^{2}$, until $S R K$ matches the hard sphere equation. Figure 1 shows the dimensionless pressure $\left(\frac{b^{2} P}{a}\right)$ as a function of the dimensionless density $(b \rho)$ for methane. In this figure, the repulsive contribution for the $S R K E O S$, i.e. $S R K_{\text {rep }}$, was obtained by subtracting $-0.53 a \rho^{2}$ from the classical cubic model.

The same procedure was applied to different normal alkanes, from ethane to n-decane, and similar curves to the one given by Figure 1 were obtained. The different terms were then averaged resulting in a value of approximately $-0.5 a \rho^{2}$, which is equal to the term obtained by Cai and Prausnitz [30]. Hence the attractive term for the free energy density (Equation 3) was set equal to:

$$
\alpha \rho^{2}=0.5 a \rho^{2}
$$

Equations 3 and 21 were then applied for the calculation of the repulsive term of the free energy density using the $S R K E o S$, and the initial term $\left(f_{0}\right)$ for the recursive procedure was determined as:

$$
f_{0}=\rho R T \ln \left(\frac{\rho}{1-\rho b}\right)-\rho \frac{a}{b} \ln (1+\rho b)+0.5 a \rho^{2}
$$

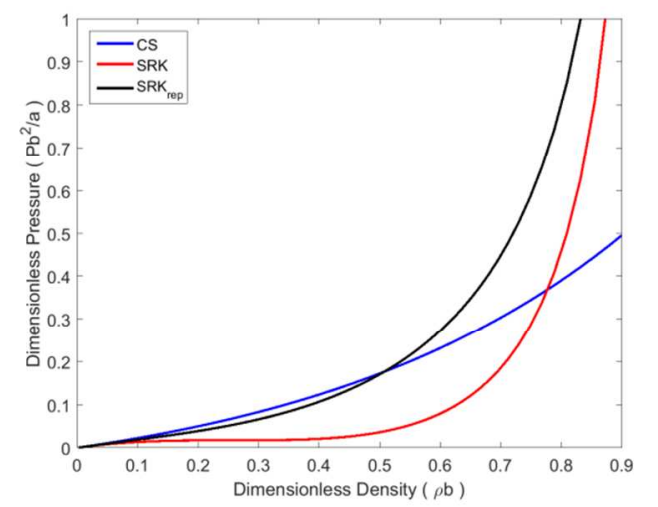

Figure 1: Dimensionless pressure $\left(\frac{b^{2} P}{a}\right)$ as a function of the dimensionless density $(b \rho)$ of methane calculated with the Carnahan-Starling EoS (CS), blue line, the SRK $E o S$, red line and the repulsive part of the cubic model $\left(S R K_{\text {rep }}\right)$, which correspond to adding the term $-0.53 a \rho^{2}$ to pressure calculated with $S R K$.

Regarding the calculation of the range of the attractive potential $(w)$ it is important to note that the parameter $\psi$ is related to the initial shortest wavelength of the density fluctuations, hence the whole term $\left(\frac{\psi w^{2}}{2 L^{2}}\right)$ in Equation 13 can be considered as an adjustable parameter $(\phi)$. With this modification, Equation 13 is given by:

$$
\bar{f}_{n}^{s}=f_{n-1}+\frac{\alpha \rho^{2} \phi}{2^{2 n}}
$$

which is the same expression proposed by Salvino and White, where $\phi$ corresponds to some multiple of the inverse square of the initial shortest wavelength of the density fluctuations [13].

The isomorphism assumption can be used in order to apply the crossover $E o S$ to mixtures [43]. This is to consider that the thermodynamic potential of a multicomponent system near its critical point has the same universal form of the pure fluid, which is valid if the chemical potentials are taken as independent variables [44]. This requirement increases the complexity of the calculations and poses an inconvenience since most the mean-field models use the mole fractions as independent variables. Kiselev and Friend [10] modified the isomorphism assumption and utilized the mole fractions as independent variables obtaining a good approximation in the description of the properties of mixtures. Furthermore, several authors have adopted this method and applied it to different $E o S$ [30] [31] [32] [33] [34] [35] [36]. 
Aside from Kiselev and Friend's approximation, the application of the crossover model to describe mixtures requires the use of mixing rules for the mean-field term and the recursion relations. For the first case, the classical van der Waals mixing rules were utilized to calculate the mixture's constants, as described by the following expressions:

$$
\begin{gathered}
a_{\text {mix }}=\sum_{i=1}^{n} \sum_{i=1}^{n} x_{i} x_{j} \sqrt{a_{i} a_{j}}\left(1-k_{i j}\right) \\
b_{\text {mix }}=\sum_{i=1}^{n} x_{i} b_{i}
\end{gathered}
$$

where $x_{i}, a_{i}$ and $b_{i}$ are the molar fraction and the attractive and repulsive term constants of component $i$, while $k_{i j}$ is a binary interaction coefficient. For the crossover term, two mixing rules were used to determine the mixture's parameters, $L_{m i x}$ and $\phi_{\text {mix }}$, which are given by:

$$
\begin{gathered}
L_{\text {mix }}^{3}=\sum_{i=1}^{n} x_{i} L_{i}^{3} \\
\phi_{\text {mix }}=\sum_{i=1}^{n} x_{i} \phi_{i}
\end{gathered}
$$

Since the parameter $L_{i}$ is the length of the threedimensional space in which the mean-field model is assumed to correctly represent the molecular interactions of component $i$, then $L_{\text {mix }}^{3}$ (Equation 26) is just the volume characterizing the density fluctuations for the mixture weighted by the molar fraction of the species in the system. Moreover, the expression for determining $\phi_{\text {mix }}$ (Equation 27) involves the mole fraction $x_{i}$ and the initial shortest wavelength of the density fluctuations for each substance $\left(\phi_{i}\right)$.

In summary, the calculation of the thermodynamic properties with the crossover SRK for pure or multicomponent starts with the determination of the initial free energy, Equation 22. Then the recursive relations, expressions 6 to 14, are applied to take into account the density fluctuations and the free energy of the system is obtained from the relation 7. After that, the thermodynamic properties are obtained by the differentiation of the Helmholtz energy.

\section{Application of the crossover $S R K$ to pure n-alkanes}

The application of the crossover $S R K$ to describe the properties of pure $\mathrm{n}$-alkanes requires the numerical integration of equations 9 and 10. Additionally, it is needed an interpolation scheme to obtain a continuous function of the free energy density corrections, Equation 7. The scheme used in this work was the cubic spline. Following other works [30] [31] [32] [33] [34] [35] [36], the density range, i.e. from $\rho=10^{-12}$ to $\rho=0.999 b^{-1}$, was divided into 500 steps, since it allows good accuracy for the calculations with the recursive procedure. Moreover, it was chosen 5 iteration steps for the inclusion of the density fluctuations in the mean-field model. Finally, the initial pressure equation was obtained from the differentiation of Equation 22 and, after applying the recursive procedure, the volume of each phase was determined with the procedure developed by Michelsen [45].

Table 1 shows the parameters obtained for the nalkanes studied in this work. The mean-field parameters, i.e. the virtual critical temperatures $\left(T_{c}^{\prime}\right)$ and pressures $\left(P_{c}^{\prime}\right)$ and acentric factors $\left(\omega^{\prime}\right)$, were regressed using saturated pressure data and saturated liquid volume data from a temperature range of $T_{r}=0.5$ to 0.75 .

\begin{tabular}{|c|c|c|c|c|c|c|c|c|}
\hline \multirow[b]{2}{*}{ Substance } & \multicolumn{5}{|c|}{ Parameters } & \multicolumn{3}{|c|}{ Experimental } \\
\hline & $T_{c}^{\prime}(\mathrm{K})$ & $P_{c}^{\prime}$ (bar) & $\omega^{\prime}(-)$ & $L(\AA)$ & $\phi(-)$ & $T_{c}(\mathrm{~K})$ & $P_{c}$ (bar) & $\omega \quad(-)$ \\
\hline $\mathrm{C} 1$ & $\begin{array}{l}197.7 \\
\end{array}$ & $\begin{array}{l}50.39 \\
\end{array}$ & -0.0578 & 4.166 & 2.50 & 190.6 & 45.99 & 0.0115 \\
\hline $\mathrm{C} 2$ & 314.3 & 52.35 & 0.0392 & 5.264 & 1.20 & 305.3 & 48.72 & 0.0995 \\
\hline $\mathrm{C} 3$ & 382.2 & 46.74 & 0.0856 & 5.913 & 0.67 & 369.8 & 42.48 & 0.1523 \\
\hline $\mathrm{C} 4$ & 441.7 & 42.48 & 0.1204 & 6.452 & 0.60 & 425.2 & 37.96 & 0.2002 \\
\hline $\mathrm{C} 5$ & 489.1 & 38.53 & 0.1632 & 6.879 & 0.60 & 469.7 & 33.70 & 0.2515 \\
\hline $\mathrm{C} 6$ & 526.4 & 34.98 & 0.2247 & 7.207 & 0.60 & 507.6 & 30.22 & 0.3013 \\
\hline $\mathrm{C} 7$ & 561.4 & 32.46 & 0.2647 & 7.396 & 0.60 & 540.2 & 27.40 & 0.3485 \\
\hline $\mathrm{C} 8$ & 592.8 & 30.17 & 0.3044 & 7.597 & 0.60 & 568.7 & 24.90 & 0.3995 \\
\hline C9 & 620.7 & 28.09 & 0.3434 & 7.809 & 0.60 & 594.6 & 22.90 & 0.4436 \\
\hline $\mathrm{C} 10$ & 645.0 & 26.24 & 0.3882 & 8.041 & 0.60 & 617.7 & 21.10 & 0.4923 \\
\hline
\end{tabular}

Table 1: Parameters of the crossover $S R K$ for methane to n-decane and experimental critical temperatures, critical pressures, and acentric factors. The parameters $T_{c}^{\prime}, P_{c}^{\prime}$ and $\omega^{\prime}$ represent the virtual critical parameters and are used in the $S_{R} K_{2} E o S$ and in the mean-field term of the crossover $S R K$. 
The reason for this approach is that the mean-field model essentially works well in regions far from the critical point, where density fluctuations do not affect the thermodynamic properties of the system. Therefore, the experimental critical parameters are changed in order to describe the correct behavior of the saturated properties far away from the critical point. Following the fitting of the mean-field parameters, the cutoff length and the initial wavelength of the short range interactions were optimized using near-critical data, i.e. saturation pressures and saturated liquid phase volume close to the critical point $\left(\mathrm{T}_{\mathrm{r}}=0.95\right.$ to 1.0$)$. White's procedure has a strong effect on the free energy calculated from the classical $E o S$ close to the critical point, changing the analytical character of the mean-field model, allowing it to capture the singularities that arise close to the critical point.

In order to assess the improvement attained with the application of the recursive procedure, Figure 2 shows the critical isotherms for n-hexane calculated with the traditional SRK EOS using the experimental critical parameters and the mean-field model with the parameters determined by fitting the experimental saturated data far away from the critical point $\left(S R K_{2}\right)$, as well as the crossover $S R K$ (CSRK). In other words, the $S R K_{2}$ model is the $S R K E O S$ with the virtual critical parameters, while the CSRK EOS is the resulting equation of the application of White's recursive procedure to the $\mathrm{SRK}_{2} E O S$.

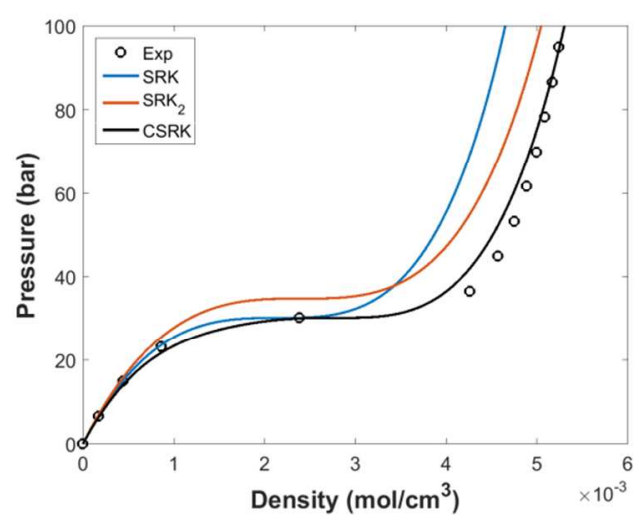

Figure 2: Critical isotherms of n-hexane calculated with the $S R K, S R K_{2}$ and CSRK EoS.

Figure 2 indicates that the use of the $E o S$ optimized to describe the behavior of the system at low reduced temperatures causes an overshoot of the critical point $\left(S R K_{2}\right)$. The application of the recursive procedure corrects this behavior and matches the experimental critical pressure. Additionally, for pressures above the critical, the description of the density with the CSRK EOS is superior to the $S R K E o S$. This is due to the fact that, far from the critical point, long-range fluctuations are no longer affecting the properties of the system and the use of parameters matching experimental saturated data enhances the description of the volume in the supercritical region.

Additionally, Table 2 shows the average absolute deviations $(A A D)$ obtained from the comparison of the experimental pressure up to $100 \mathrm{bar}$ at the critical isotherm of n-hexane and the values calculated at the critical isotherm by the SRK, SRK 2 and CSRK EOS. As observed in Figure 2, it is possible to conclude that the change in the parameters of the SRK EOS reduces the deviations in terms of pressure in more than one half, mainly due to the better description of the supercritical region. Nevertheless, the errors are approximately $30 \%$. On the other hand, the application of the crossover procedure to the mean-field model improves the representation of this property ( $A A D$ is close to $9 \%$ ), primarily near the critical point where the fluctuations are affecting the behavior of the system. Although this comparison is done only for n-hexane, similar behavior is seen for the other n-alkanes studied in this work.

Table 2: $A A D$ of pressures up to $100 \mathrm{bar}$ for the critical isotherms of n-hexane calculated with the $S R K, S R K_{2}$ and CSRK EOS

\begin{tabular}{|c|c|c|c|}
\hline & SRK & $S_{R} K_{2}$ & CSRK \\
\hline$\Delta P(\%)$ & 67.4 & 30.6 & 8.79 \\
\hline
\end{tabular}

The three models were also applied in the calculation of the saturation properties of the n-alkanes studied in this work. Figure 3a shows the saturation pressure as a function of temperature and Figure $3 \mathrm{~b}$ shows the temperature as a function of the density of the coexisting phases for selected normal alkanes (methane, propane, $\mathrm{n}$ hexane, and n-decane).

The main features of each model can be observed in Figure 3. In the case of the mean-field equations, it can be seen that $S R K$ is capable of correctly describing the vapor pressures from low temperatures to the critical point; nevertheless, it gives large deviations with respect to the density of the saturated liquid phase. One way to improve this behavior is to use volume translation correlations, but such procedures are usually applied for regions far from the critical point, in which the density fluctuations do not affect the properties of the system [46] [47]. 


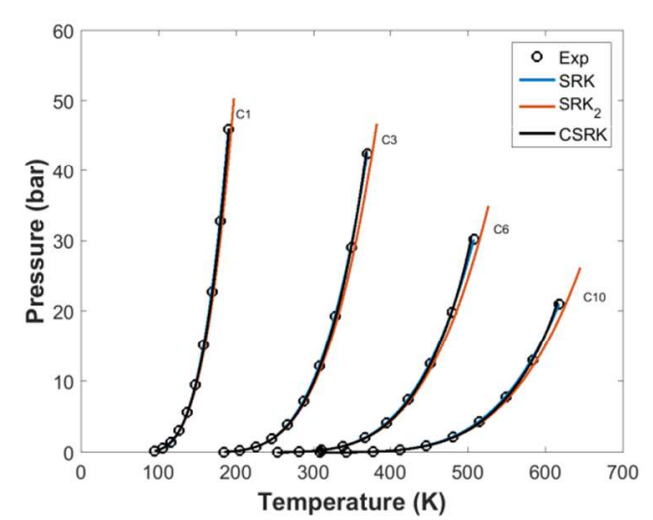

(a)

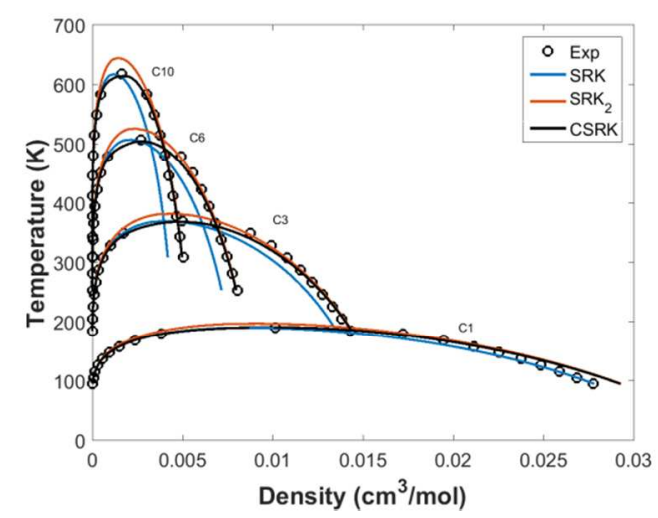

(b)

Figure 3: Saturation pressure as a function of temperature (a) and temperature as a function of the coexisting phases density (b) for selected n-alkanes (from methane to ndecane). Experimental data (open circles) taken from the NIST database, while the blue line corresponds to the $S R K E O S$, the red line corresponds to the $S R K_{2} E O S$ and the black line corresponds to the CSRK EoS.

Another alternative is to use parameters regressed from experimental saturated data $\left(S R K_{2}\right)$, as shown in the figures by the red line. In this approach, the description of the liquid density is improved, in comparison to the classical SRK model; nonetheless, it over-predicts the critical point and reduces the accuracy of the description of the vapor pressure of the pure fluids. A similar behavior was observed by Vinhal et al. [48] with the use of the Cubic-Plus-Association $(C P A) E O S$ [49]. The $C P A$ $E O S$ is a combination of the $S R K$ model and the association term from Wertheim's theory [50], in which the cubic $E o S$ takes into account the physical interactions between molecules, while the association term accounts for the hydrogen bonding and other interactions between molecules [49]. For hydrocarbons, $C P A$ reduces to $S R K$ and the superior description of the liquid phase density is related to the parameterization procedure, i.e. fitting experimental saturation pressures and liquid phase densities from a reduced temperature range of 0.5 to 0.9 or 0.95 [51]. Additionally, rescaling the parameters for improving critical point representations increases the deviation of the saturated liquid phase volume, since mean-field models are not capable of describing the nonanalytical behavior in the near-critical region [48].

The deficiencies in the $S R K_{2}$ model can be corrected with the utilization of the recursive procedure, as shown in the figures by the black line. The crossover $S R K E O S$ $(C S R K)$ corrects the overprediction of the $S R K_{2}$ model, matching the experimental critical temperatures and pressures for the selected hydrocarbons, as well as precisely describes the saturated liquid densities.

In addition to the qualitative comparison of the models, it is also important, for engineering applications, to evaluate the quantitative predictions of the saturated properties. Table 3 shows the absolute average deviations $(A A D)$ for the vapor pressure and the molar volume of the coexisting phases calculated with the aforementioned models in the reduced temperature range of 0.5 to 0.95 . It also shows the deviations for the CPA EOS, with the parameters taken from [52]. As seen previously, the classical SRK accurately describes the vapor pressure of the normal alkanes, besides it gives an excellent description of the volume of the vapor phase. From the table, it is observed that the deviations of the molar volume increase with the carbon number of the hydrocarbon. The use of parameters regressed from experimental saturated data $\left(S R K_{2}\right)$ improves the description of the saturated liquid phase volume, but deteriorates the representation of the other properties, especially for the molar volume of the saturated vapor phase. Furthermore, the CPA EOS provides better descriptions of the saturated properties due to the wider range of reduced temperature used in the parametrization procedure. The crossover SRK gives similar representations in comparison with $C P A$, although the deviations in the saturated pressure and liquid phase volume are higher.

A complete evaluation of the models requires an assessment of the performance of the EoS in the description of the properties near the critical point. Therefore, the critical temperatures, pressures, and volumes of the normal alkanes were calculated and compared to the experimental values. Table 4 shows the $A A D$ for the critical properties obtained for each model. Since the SRK EOS was designed to match the experimental critical temperature and pressure of a component, the error is zero. 
Table 3: $A A D$ of the saturation pressure and volumes of the vapor and liquid phase for methane to n-decane, as well as the average of all deviations, calculated using the $S R K E O S$, the $S R K_{2} E O S$ and the CSRKEOS. The reduced temperature range of calculations was from 0.5 to 0.95 .

\begin{tabular}{|c|c|c|c|c|c|c|c|c|c|c|c|c|}
\hline \multirow{2}{*}{ Substance } & \multicolumn{4}{|c|}{$\Delta P^{\text {sat }}(\%)$} & \multicolumn{4}{|c|}{$\Delta v^{l i q}(\%)$} & \multicolumn{4}{|c|}{$\Delta v^{v a p}(\%)$} \\
\hline & SRK & $S R K_{2}$ & $C P A$ & CSRK & $S R K$ & $\mathrm{SRK}_{2}$ & $C P A$ & CSRK & SRK & $S R K_{2}$ & $C P A$ & CSRK \\
\hline $\mathrm{C} 1$ & 2.21 & 3.81 & 0.65 & 1.73 & 4.07 & 3.60 & 2.49 & 3.22 & 2.44 & 6.75 & 1.59 & 2.74 \\
\hline $\mathrm{C} 2$ & 1.72 & 3.78 & 0.24 & 1.57 & 7.04 & 1.25 & 1.95 & 2.15 & 1.80 & 6.70 & 2.18 & 3.05 \\
\hline $\mathrm{C} 3$ & 1.39 & 4.34 & 2.07 & 2.03 & 8.64 & 1.00 & 2.02 & 1.86 & 1.33 & 7.46 & 3.66 & 3.41 \\
\hline $\mathrm{C} 4$ & 1.55 & 4.85 & 0.35 & 2.52 & 10.1 & 1.17 & 3.55 & 1.70 & 1.39 & 8.51 & 2.94 & 3.34 \\
\hline $\mathrm{C} 5$ & 1.54 & 5.46 & 0.33 & 3.28 & 12.3 & 0.50 & 1.32 & 1.39 & 1.54 & 9.45 & 2.61 & 2.74 \\
\hline C6 & 1.59 & 4.32 & 1.80 & 2.30 & 14.3 & 0.61 & 0.91 & 1.59 & 1.94 & 8.36 & 5.05 & 2.39 \\
\hline $\mathrm{C} 7$ & 1.30 & 4.25 & 0.70 & 2.43 & 16.1 & 0.74 & 0.83 & 1.25 & 1.65 & 8.58 & 4.07 & 2.66 \\
\hline C8 & 1.41 & 4.68 & 0.81 & 2.94 & 18.0 & 1.22 & 0.81 & 1.06 & 1.33 & 8.79 & 2.65 & 2.63 \\
\hline C9 & 1.58 & 4.73 & 0.85 & 3.09 & 19.5 & 1.27 & 0.83 & 1.06 & 0.95 & 9.01 & 2.85 & 2.25 \\
\hline $\mathrm{C} 10$ & 2.08 & 4.64 & 2.45 & 3.11 & 20.7 & 1.69 & 0.83 & 0.94 & 2.17 & 9.52 & 1.51 & 1.92 \\
\hline Average & 1.64 & 4.49 & 1.03 & 2.50 & 13.1 & 1.31 & 1.55 & 1.62 & 1.65 & 8.31 & 2.91 & 2.71 \\
\hline
\end{tabular}

However, in the case of the critical volume, the deviations increase considerably with the molecular weight of the hydrocarbon. An improvement in the representation is obtained with the use of different parameters for the mean-field model $\left(S R K_{2}\right.$ and $\left.C P A\right)$; nonetheless, as seen for the saturation properties this approach increases the deviations for the other critical properties. The CSRK EOS corrects this behavior enhancing the representation of the critical properties. This is observed in Table 4 since the crossover model precisely describes the critical properties, although a small increase in the deviations of the critical pressures and temperatures is obtained in comparison to the traditional SRK EOS.

\section{Application of the crossover SRK to binary methane/n-alkane mixtures}

With the aim to further assess the performance of the $E o S$, phase equilibrium and critical point calculations were performed for different binary methane and nalkane mixtures (methane/ethane to methane/n-decane). The choice of such systems was done due to the fact that methane/n-alkane mixtures show a continuous transition of phase behavior that is representative of hydrocarbon family [53]. Systems containing methane and a light hydrocarbon present a type I behavior, according to van Konynenburg and Scott [54] classification and as the molecular weight of the heavier compound increases (nheptane of heavier alkanes), they are part of the type III group. The prediction of the continuous transition of the phase behavior of methane/n-alkane systems is important in the analysis of the models.

In this section, we compare the results for vaporliquid equilibrium $(V L E)$ and critical properties obtained with the SRK, SRK 2 and CSRK EOS. As shown in the previous section, $S R K_{2}$ and $C P A$ behave similarly, although the latter model provides better representations of the pure component properties due to the wider range of temperatures used in the parametrization procedure. Nonetheless, for the binary systems studied, similar results are obtained and the comparison of the models is only done with the $S R K_{2} E o S$.

Figure 4 shows the vapor-liquid equilibrium (VLE) diagram for the methane/ethane system for three different temperatures $(200 \mathrm{~K}, 250 \mathrm{~K}$, and $280 \mathrm{~K})$. From the plot, it is seen that the region of vapor-liquid coexistence becomes narrower as the temperature increases; besides, the amount of methane in the vapor phase reduces. For such simple symmetric systems, it is possible to observe that the SRK EOS is capable of precisely predicting the pressures and compositions of the phases without the use of a binary interaction coefficient (Figure 4a). On the other hand, the utilization of parameters regressed from pure component saturation data $\left(S R K_{2}\right)$ modify the behavior of the mean-field model, increasing the deviations from experimental data, especially for higher temperatures close to the critical point, as shown in the Figure $4 \mathrm{~b}$. The application of the recursive procedure corrects this behavior and an analogous representation compared to the $S R K E O S$ is obtained (Figure 4c).

A similar plot is given in Figure 5, which exhibits the $V L E$ diagram for the methane/n-heptane system at $311 \mathrm{~K}$, $444 \mathrm{~K}$, and $511 \mathrm{~K}$. This plot shows that the increase in the asymmetry of the systems reduces the accuracy of the models. In fact, for quantitative description of the phase equilibrium properties, the use of $k_{i j}$ is necessary. Moreover, Figure $5 \mathrm{c}$ shows that the introduction of the density fluctuations corrects the behavior of the $\mathrm{SRK}_{2}$ model (Figure 5b) and a similar description for the classical SRK is attained (Figure 5a). 
Table 4: $A A D$ of the critical temperatures, pressures and volumes for methane to $\mathrm{n}$-decane, as well as the average value of the deviations, calculated using the $S R K$, the $S R K_{2}$ and the CSRK EOS.

\begin{tabular}{|c|c|c|c|c|c|c|c|c|c|c|c|c|}
\hline \multirow{2}{*}{ Substance } & \multicolumn{4}{|c|}{$\Delta T_{c}(\%)$} & \multicolumn{4}{|c|}{$\Delta P_{c}(\%)$} & \multicolumn{4}{|c|}{$\Delta v_{c}(\%)$} \\
\hline & $S R K$ & $S R K_{2}$ & $C P A$ & CSRK & $S R K$ & $S R K_{2}$ & $C P A$ & CSRK & SRK & $\mathrm{SRK}_{2}$ & $C P A$ & CSRK \\
\hline $\mathrm{C} 1$ & 0.00 & 3.74 & 1.37 & 0.38 & 0.00 & 9.57 & 3.98 & 0.25 & 16.5 & 10.3 & 13.5 & 0.88 \\
\hline $\mathrm{C} 2$ & 0.00 & 2.94 & 1.59 & 0.02 & 0.00 & 7.45 & 6.90 & 1.00 & 19.4 & 14.4 & 13.4 & 4.87 \\
\hline $\mathrm{C} 3$ & 0.00 & 3.34 & 2.38 & 0.26 & 0.00 & 10.0 & 11.0 & 0.94 & 20.6 & 13.3 & 11.2 & 1.76 \\
\hline C4 & 0.00 & 3.90 & 2.63 & 0.33 & 0.00 & 12.9 & 14.9 & 0.94 & 21.7 & 12.0 & 8.75 & 1.20 \\
\hline C5 & 0.00 & 4.13 & 2.08 & 0.19 & 0.00 & 14.3 & 12.6 & 1.21 & 23.4 & 12.4 & 11.9 & 1.45 \\
\hline C6 & 0.00 & 3.70 & 2.90 & 0.56 & 0.00 & 15.6 & 15.3 & 1.40 & 25.4 & 12.4 & 11.9 & 1.89 \\
\hline C7 & 0.00 & 3.92 & 2.59 & 0.59 & 0.00 & 18.5 & 16.2 & 1.25 & 27.7 & 12.0 & 12.7 & 3.21 \\
\hline $\mathrm{C} 8$ & 0.00 & 4.24 & 2.44 & 0.46 & 0.00 & 21.2 & 18.3 & 1.48 & 30.2 & 12.0 & 12.8 & 3.92 \\
\hline $\mathrm{C} 9$ & 0.00 & 4.39 & 2.62 & 0.41 & 0.00 & 22.7 & 19.7 & 1.01 & 30.3 & 10.8 & 11.7 & 5.51 \\
\hline $\mathrm{C} 10$ & 0.00 & 4.42 & 2.16 & 0.35 & 0.00 & 24.4 & 20.6 & 1.50 & 33.1 & 11.7 & 12.7 & 5.07 \\
\hline Average & 0.00 & 3.88 & 2.28 & 0.35 & 0.00 & 15.7 & 13.9 & 1.10 & 24.8 & 12.1 & 12.1 & 2.98 \\
\hline
\end{tabular}

In order to improve the description of the $V L E$ systems as shown, in Figures 4 and 5, binary interaction coefficients $\left(k_{i j}\right)$ were regressed from experimental phase equilibrium data. The calculation of $k_{i j}$ were done by minimizing the objective function $(O . F$.$) represented$ by the equation:

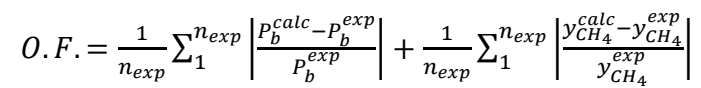

where $P_{b}$ is the bubble point pressure and $y_{C_{4}}$ is the composition of methane in the vapor phase, the subscripts calc and exp represent calculated and experimental values, respectively, and $n_{\text {exp }}$ is the number of experimental points.

Table 5 shows the average $A A D$ for all the binary methane/n-alkane systems studied in this work for different temperatures. The bubble point pressures $\left(\Delta P_{b}\right)$ and the composition of methane in the vapor phase $\left(\Delta y_{C_{4}}\right)$ were calculated with the optimum $k_{i j}$ values and compared to the experimental data given by the reference in the table. The results show that the three models correctly correlate the bubble point pressure. Nevertheless, the CSRK EOS is superior to the two approaches of the mean-field model.

In the case of the deviations for the composition of methane in the vapor phase, the classical SRK EoS is slightly better than the CSRK model. In fact, the improvement in the description of the composition of methane in the vapor phase with the $S_{R K} K_{2} E S$ by applying the recursive procedure is substantial. This is due to correction of the mean-field model close to the critical point.
The evaluation of the binary interaction coefficients in Table 4 shows that the $S R K$ model requires smaller $k_{i j}$ values for correlating VLE data in comparison to the other equations. On the other hand, the optimized $k_{i j}$ for the $S_{R K}$ and $C S R K$ are similar. Since most of the experimental points were far from the critical region, this indicates that the two models behave equivalently in such regions and the improvements in the representations are mainly due to the introduction of density fluctuations into the mean-field model.

In addition to the $V L E$ representations, critical line predictions were performed for the systems described previously and compared to experimental data [65]. The algorithm for calculating the critical points is given in [48].

Figure 6 shows the critical pressures as a function of the critical temperatures for the systems: methane/ethane, methane/propane, and methane/n-butane. The three mixture possess a type I behavior, for which the critical line connects the two pure component critical points. The graph indicates that the curves obtained with SRK correctly describe the experimental data, while $S R K_{2}$ over-predicts almost all the measured critical points. The reason for this behavior is due to the use of virtual critical parameters that changes the prediction of the pure components critical point. The introduction of density fluctuations in the second mean-field approach allows a better representation of the experimental data. Moreover, the $k_{i j}$ values obtained for the $V L E$ descriptions were not used in the critical point calculations since they increased the deviations from experimental critical data. 
Table 5: Average AAD of the bubble point pressure and composition of methane in the vapor phases for binary methane/nalkane systems (methane/ethane to methane/n-decane) for several temperatures calculated using the $S R K$, the $S R K_{2}$ and CSRK EOS.

\begin{tabular}{|c|c|c|c|c|c|c|c|c|c|c|c|}
\hline \multirow{2}{*}{ System } & \multicolumn{3}{|c|}{$\Delta P_{b}(\%)$} & \multicolumn{3}{|c|}{$\Delta y_{\mathrm{CH}_{4}}(\%)$} & \multicolumn{3}{|c|}{$k_{i j}$} & \multirow{2}{*}{$\mathrm{T}(\mathrm{K})$} & \multirow{2}{*}{ Reference } \\
\hline & SRK & $\mathrm{SRK}_{2}$ & CSRK & SRK & $\mathrm{SRK}_{2}$ & $C S R K$ & SRK & SRK2 & CSRK & & \\
\hline $\mathrm{C} 1-\mathrm{C} 2$ & 1.81 & 4.35 & 2.09 & 2.30 & 7.81 & 2.77 & 0.0029 & 0.0406 & 0.0347 & $\begin{array}{l}200 \\
250 \\
280\end{array}$ & {$[55][56]$} \\
\hline $\mathrm{C} 1-\mathrm{C} 3$ & 1.40 & 3.09 & 1.30 & 8.14 & 9.51 & 10.2 & 0.0196 & 0.0473 & 0.0502 & $\begin{array}{l}280 \\
328 \\
361\end{array}$ & [57] \\
\hline $\mathrm{C} 1-\mathrm{C} 4$ & 2.86 & 3.77 & 2.97 & 6.33 & 14.7 & 7.30 & 0.0366 & 0.0592 & 0.0581 & $\begin{array}{l}244 \\
344 \\
410\end{array}$ & [58] [59] \\
\hline $\mathrm{C} 1-\mathrm{C} 5$ & 2.58 & 3.02 & 2.32 & 3.47 & 7.04 & 2.56 & 0.0234 & 0.0426 & 0.0456 & $\begin{array}{l}278 \\
378 \\
411\end{array}$ & [60] \\
\hline C1-C6 & 5.86 & 4.36 & 5.40 & 3.56 & 5.92 & 2.82 & 0.0136 & 0.0324 & 0.026 & $\begin{array}{l}311 \\
378 \\
444\end{array}$ & [61] \\
\hline $\mathrm{C} 1-\mathrm{C} 7$ & 3.41 & 3.35 & 2.01 & 4.04 & 7.13 & 3.88 & 0.0222 & 0.0394 & 0.0368 & $\begin{array}{l}311 \\
444 \\
510\end{array}$ & [62] \\
\hline $\mathrm{C} 1-\mathrm{C} 8$ & 2.30 & 1.08 & 1.46 & 0.37 & 0.16 & 0.36 & 0.0409 & 0.0490 & 0.0499 & $\begin{array}{l}298 \\
348 \\
423\end{array}$ & [63] \\
\hline C1-C9 & 2.52 & 3.85 & 2.80 & 0.57 & 0.50 & 0.52 & 0.0363 & 0.0433 & 0.0397 & $\begin{array}{l}298 \\
348 \\
423\end{array}$ & [63] \\
\hline $\mathrm{C} 1-\mathrm{C} 10$ & 2.96 & 5.10 & 3.49 & 0.74 & 2.45 & 1.19 & 0.0409 & 0.0287 & 0.0315 & $\begin{array}{l}311 \\
444 \\
510\end{array}$ & [64] \\
\hline Average & 2.86 & 3.55 & 2.65 & 3.28 & 6.13 & 3.51 & - & - & - & - & - \\
\hline
\end{tabular}

The increase in the molecular weight of the hydrocarbon changes the behavior of the critical lines in comparison with the previous systems. For the methane/n-pentane system, Figure 7 a shows that all the models, except for $S R K$, predict a type I behavior. The $S R K E O S$ predicts a type $\mathrm{V}$ behavior, which means that the two critical pure critical points are no longer connected by a line. In fact, the critical line going from the heavier component ends on a lower critical endpoint (LCEP), due to a split of the liquid phase into two liquids. This three-phase line is connected to an upper critical endpoint (UCEP), which is linked to the critical point of the light species. Experimental data suggests that there might be a region of liquid-liquid immiscibility close to the critical point of pure methane; nonetheless, this point has not been detected, thus both of the critical lines are correct to represent the system's behavior [53]. The models predicted a Type $\mathrm{V}$ behavior for binary systems containing species heavier than n-pentane (Figure 7b).

With respect to the predictions of the experimental critical pressures and temperatures, for both the methane/n-pentane and methane/n-heptane systems, it is seen in Figure 8 that the CSRK is superior to the other models, as it corrects the description of the $\mathrm{SRK}_{2} \mathrm{EoS}$, especially in regions with high concentration of the heavier hydrocarbons, besides it has a lower maximum critical pressure in comparison to the SRK EOS. 


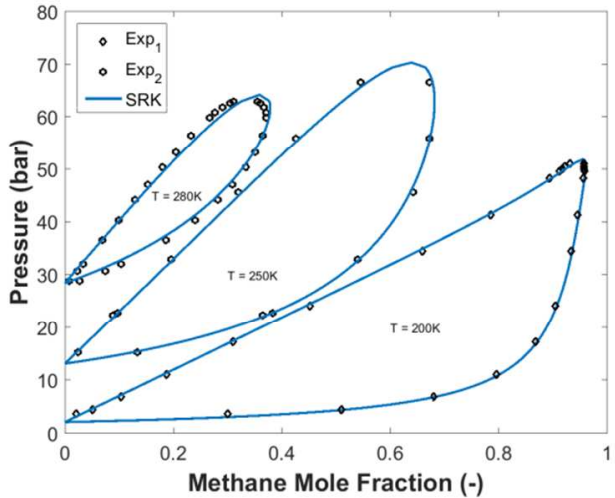

(a)

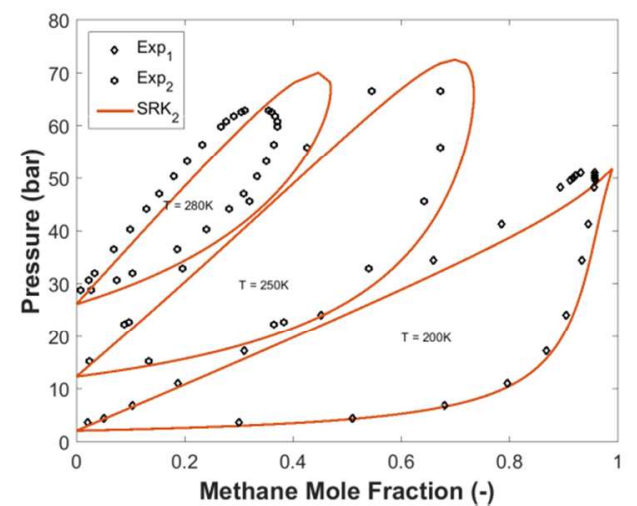

(b)

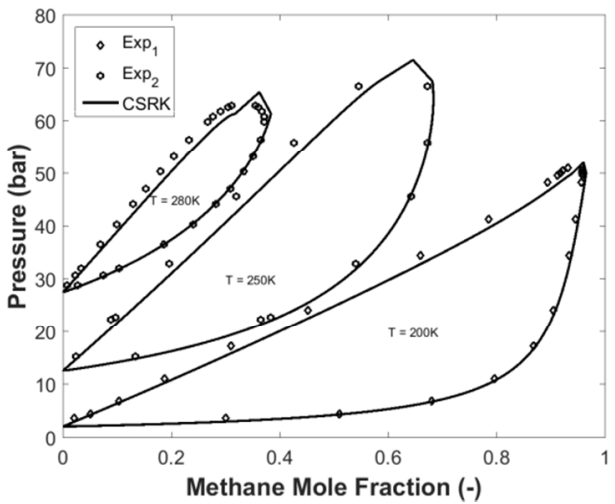

(c)

Figure 4: Experimental VLE diagram of the methane/ethane (open circles and diamonds) and the predictions $\left(k_{i j}=0\right)$ using the $S R K \operatorname{EoS}(\mathrm{a})$, the $S R K_{2}$ $E O S$ (b) and the CSRK EOS (c) for three different temperatures. The experimental data $\operatorname{Exp}_{1}$ and $\operatorname{Exp}_{2}$ were taken from [55] and [56], respectively.

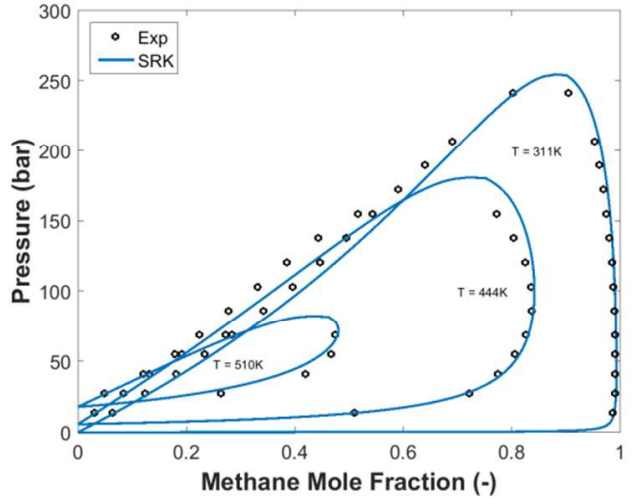

(a)

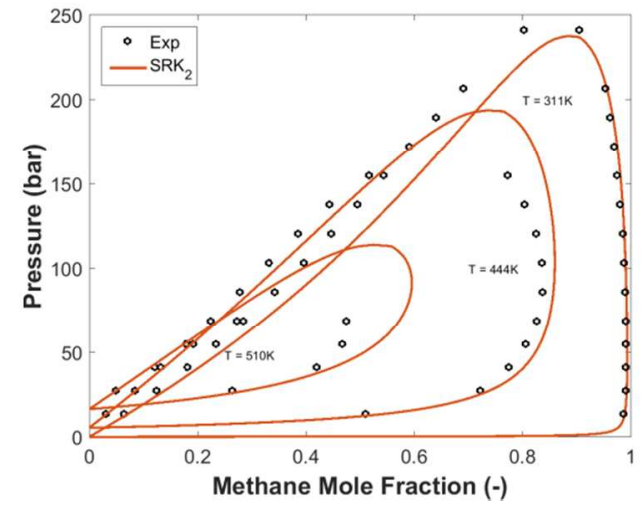

(b)

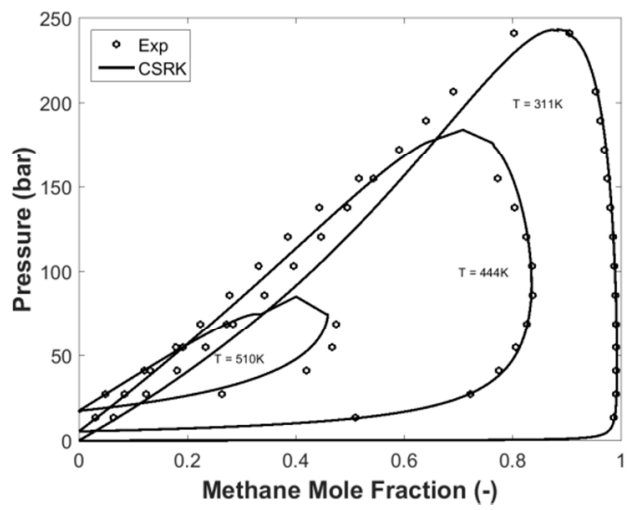

(c)

Figure 5: Experimental $V L E$ diagram of the methane/nheptane (open circles) and the predictions $\left(k_{i j}=0\right)$ using the $S R K \operatorname{EoS}(\mathrm{a})$, the $S R K_{2} \operatorname{EoS}(\mathrm{b})$ and the CSRK EOS (c) for three different temperatures. The experimental data was taken from [62]. 


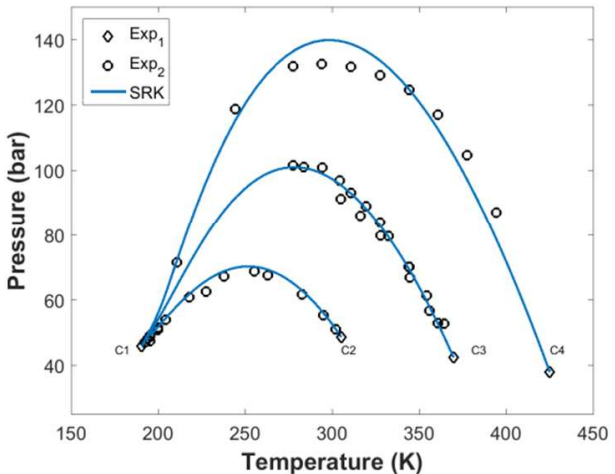

(a)

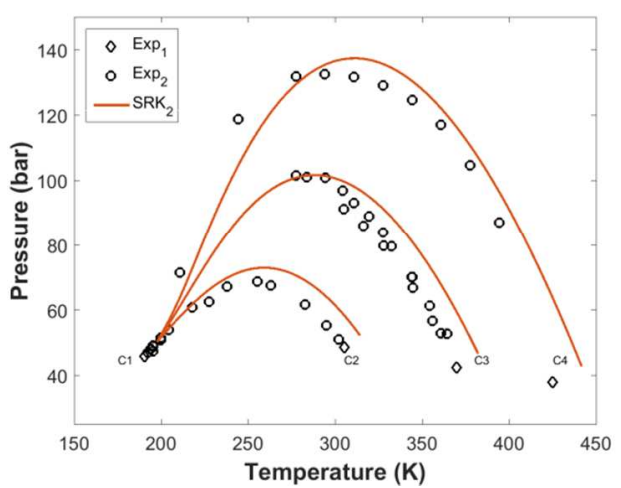

(b)

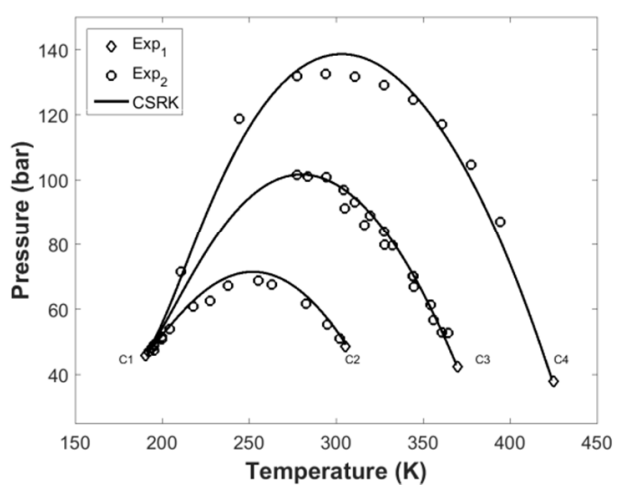

(c)

Figure 6: Experimental critical temperature as function of the critical pressure for the systems methane/ethane, methane/propane and methane/n-butane (open circles and diamonds) and the predicted critical lines $\left(k_{i j}=0\right)$ using the $S R K \operatorname{EoS}$ (a), the $S R K_{2} \operatorname{EoS}$ (b) and the CSRK EoS (c) for three different temperatures. The experimental data were taken from [65].

Figure 8 shows the critical pressures as a function of the composition of methane for the previous systems. In this graph, it is easier to observe the behavior of the system close to of pure methane. As discussed before, only the SRK EoS predicts a type $\mathrm{V}$ behavior for the methane/n-pentane system (Figure 8a), while the other models predict a type I behavior with a sharp variation in composition close to pure methane [53]. For the methane/n-heptane system, the three equations have similar features (Figure $8 \mathrm{~b}$ ), predicting a type $\mathrm{V}$ behavior, which is represented by a discontinuity in the critical line. Moreover, due to the appearance of a second liquid, the system is no longer represented by the fluctuations in density. As a matter of fact, the order parameter becomes the composition of one the components, thus a different method should be applied in order to incorporate the fluctuations into to the mean-field model [66]. The comparison of the experimental data with the calculated curves shows that the SRK2 and CSRK EoS describe the critical pressure-composition relation similarly, while the SRK EoS predicts a higher maximum critical pressure.

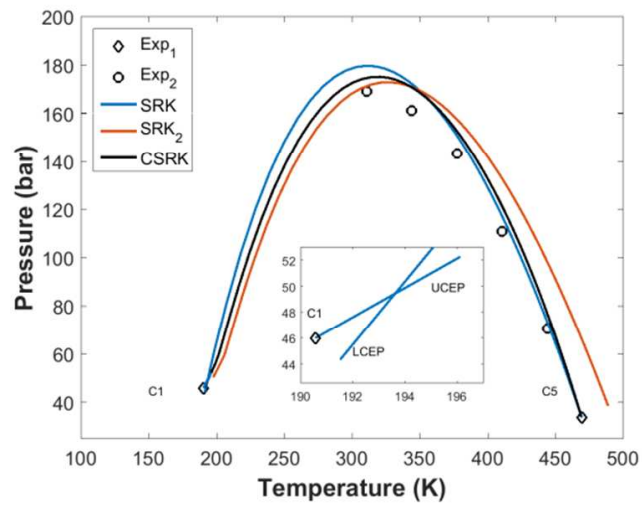

(a)

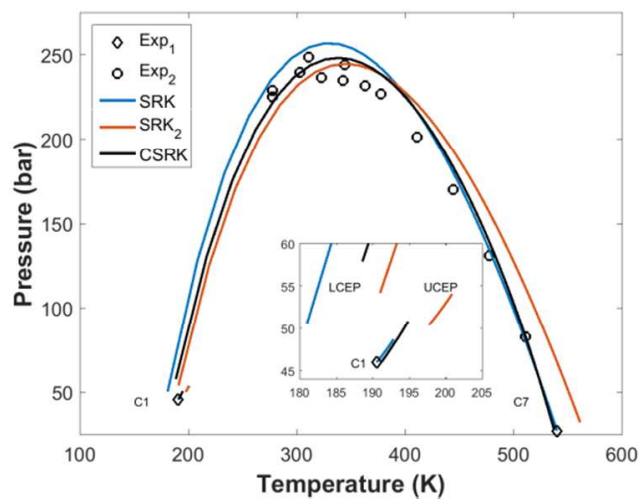

(b)

Figure 7: Experimental critical temperature as function of the critical pressure for the systems methane/n-pentane (a), methane/n-heptane (b) (open circles and diamonds) and the predicted critical lines $\left(k_{i j}=0\right)$ using the $S R K$ $E o S$ (blue line), the $S R K_{2} E o S$ (red line) and the CSRK $\operatorname{EoS}$ (black line). Experimental data were taken from [65]. 
In addition to the plots of the critical pressure as a function of composition, it was also evaluated the relationship between the critical volume and the mole fraction of the light component (Figure 9). In the figures, it is possible to see that the predictions are similar to the region of high concentration of methane; nonetheless, with the increase in the composition of the heavier nalkane, the mean-field models predict a much higher critical molar volume for the mixture in relation to the experimental data. On the other hand, the CSRK EOS gives an accurate description of the densities for the two systems.

Table 6 shows the average value of the $A A D$ for the critical properties of the methane/n-alkane binary mixtures. It indicates that the CSRK EOS is superior to the other models in predicting the critical properties, especially the critical volume, in which an improvement of approximately $12 \%$ and $3 \%$ was obtained in comparison to the $S R K$ and $S R K_{2} E O S$, respectively. Furthermore, the errors increase drastically for the systems with methane and n-hexane or heavier hydrocarbons. The reason for that is due to the fact that most experimental data were in the region with high methane concentration, in which the critical properties are affected by the fluctuations in the mole fraction of the species and none of the models take that into account.

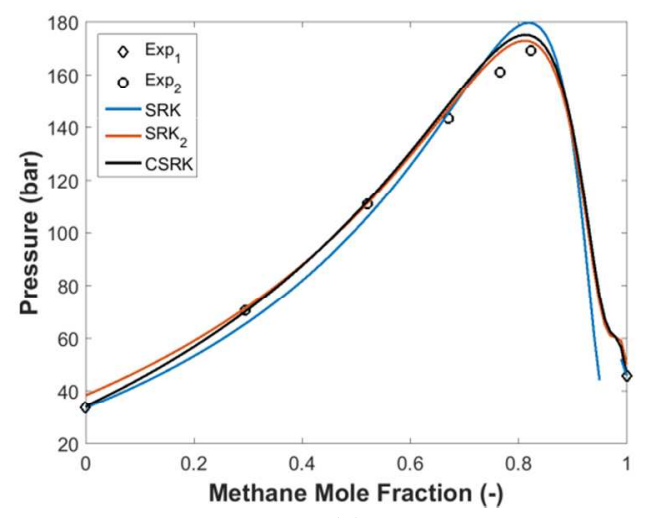

(a)

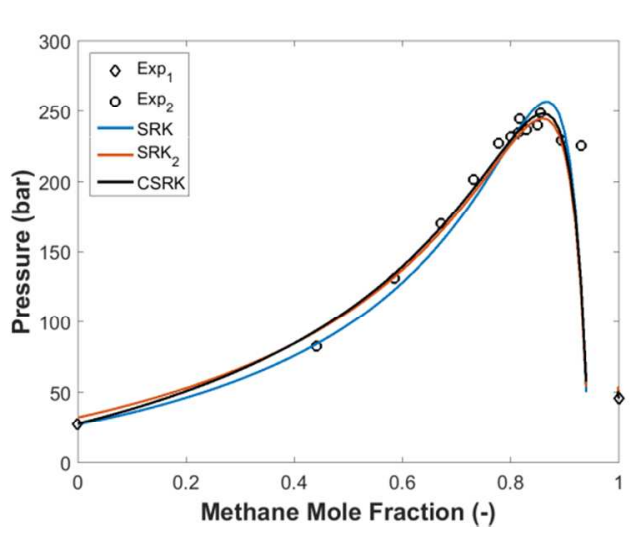

(b)

Figure 8: Experimental critical pressure as function of the methane mole fraction for the systems methane/n-pentane (a), methane/n-heptane (b) (open circles and diamonds) and the predicted critical lines ( $\left.k_{i j}=0\right)$ using the $S R K \operatorname{EoS}$ (blue line), the $S R K_{2} E O S$ (red line) and the CSRK EoS (black line). Experimental data were taken from [65].

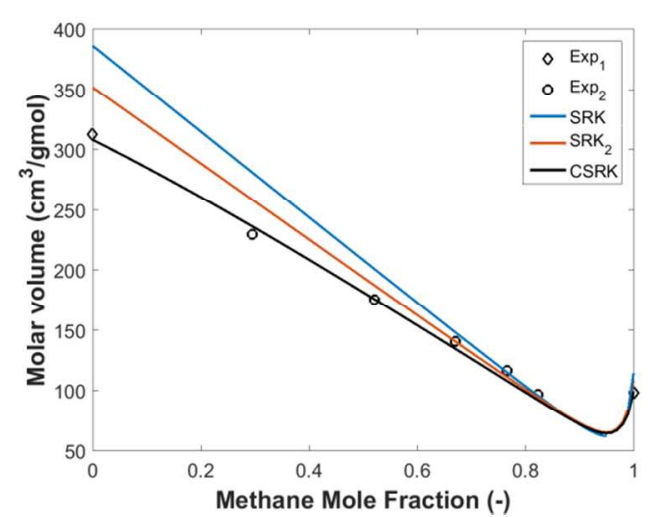

(a)

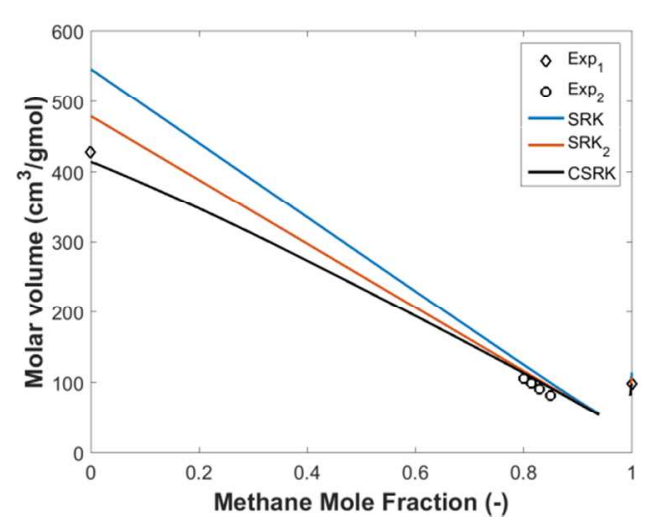

(b)

Figure 9: Experimental critical molar volume as function of the methane mole fraction for the systems methane/n-pentane (a), methane/n-heptane (b) (open circles and diamonds) and the predicted critical lines $\left(k_{i j}=0\right)$ using the $S R K \operatorname{EoS}$ (blue line), the $S_{R K} K_{2} \operatorname{EoS}$ (red line) and the CSRK EoS (black line). Experimental data were taken from [65]. 


\section{Conclusions}

The application of White's recursive procedure to the SRK EoS corrects the behavior of the model in the critical region, allowing precise description of the thermodynamic properties of pure n-alkanes (methane to n-decane) far and close to the critical point. The resulting crossover SRK (CSRK) model is also capable of accurately representing the phase equilibrium properties and improving the prediction of the critical properties of binary methane/n-alkane mixtures (methane/ethane to methane/n-decane).

The comparison of results for the pure fluids using the CSRK EoS with the two approaches of the mean-field equation, one using the experimental critical parameters (SRK) and the other using parameters obtained by matching saturation pressure and the liquid phase densities $\left(S R K_{2}\right)$, showed that the recursive procedures correct the second approach and enhances the prediction near the critical point, while maintaining the same representation of the liquid phase density far from the critical point.

In the case of $V L E$ calculations for the binary systems, the performance of the CSRK EOS is similar to $S R K$. Nevertheless, there is an improvement in the description of the bubble point pressure and the composition of methane in the vapor phase in comparison to the $S R K_{2} E o S$, which is due to the introduction of long-range interactions in the mean-field model, since far away from the critical point both equations behave similarly.
Finally, similar representations of the critical pressures and temperatures were obtained for the SRK and the CSRK EoS, although the latter model presented a slightly superior performance. Additionally, the use of the crossover model improves the predictions of the critical volume of the binary methane/n-alkane systems.

\section{Acknowledgment}

The authors would like to express their gratitude to the program "Science without Borders" for the $\mathrm{PhD}$ scholarship of Andre Pinto Coelho Muniz Vinhal.

\section{List of symbols}

Abbreviations
AAD: average absolute deviation $\left(\frac{100}{N_{\text {exp }}} \sum\left|\frac{x_{\text {calc }}-x_{\text {exp }}}{x_{\text {exp }}}\right|\right)$
BWR: Benedict-Webb-Rubin equation of state
$C P A$ : Cubic-Plus-Association equation of state
CSRK: Crossover Soave-Redlich-Kwong equation of state
EoS: equation of state
GERG: Groupe Europeen de Recherchers Gazieres
LPG: liquefied petroleum gas
NIST: National Institute of Standards and Technology
$R G:$ Renormalization Group Theory
SAFT: Statistical Associating Fluid Theory
SRK: Soave-Redlich-Kwong equation of state
VLE: Vapor-liquid equilibrium




\section{Roman letters}

$a$ : Energy parameter in an EoS

$b$ : size-related parameter in an EoS

$f$ : free energy density

$F$ : free energy functional

$G$ : functions of the modified free energy density

$k_{b}$ : Boltzmann constant

$k_{i j}$ : binary interaction coefficient

$K$ : recursive procedure coefficient

$L$ : cutoff length

$n$ : mole number

$n_{\text {exp }}$ : number of experimental points

$P$ : pressure

$R$ : ideal gas constant

$r$ : molecular distance

$T$ : temperature

$u$ : pair potential

$v$ : molar volume

$x$ : mole fraction

$w$ : range of the attractive potential

$y$ : vapor phase mole fraction

$Z$ : compressibility factor

\section{Greek letters}

$\alpha$ : interaction volume

$\delta f$ : free energy density correction

$\eta$ : packing ratio

$\kappa$ : function of the acentric facor

$\rho$ : molar density

$\varphi$ : initial shortest wavelength

$\psi$ : parameter related to the initial shortest wavelength

$\omega$ : acentric factor

$\Omega$ : contributions of the density fluctuations

\section{Super/subscripts}

att: attractive term of the pair potential

$c$ : critical property

calc: calculated property

exp: experimental property

$i, j$ : component index

mix: mixture constant

$n$ : iteration index

$l$ : long-range interactions

$r$ : reduced property

rep: repulsive part of the pair potential

$s$ : short-range interactions

vap: vapor

\section{Bibliography}

[1] Knez, Z.; Markocic, E.; Leitgeb, M.; Primozic, M.; Hrncic, M. K.; Skerget, M. Industrial Applications of Supercritical Fluids: a Review. Energy 2014, 77,
235-243.

[2] Ma, T.; Imai, H.; Suehiro, Y.; Chen, C.; Kimura, T.; Asaoka, S.; Li, X. Selective Synthesis of Gasoline from Syngas in Near-Critical Phase. Catalysis Today 2014, 228, 167-174.

[3] Zhang, Q.; Liu, P.; Fujiyama, Y.; Chen, C.; Li, X. Synthesis of light hydrocarbons from syngas in near-critical phase. Applied Catalysis A: General 2011, 401, 147-152.

[4] Liu, J.; Qin, Z.; Wang, G.; Hou, X.; Wang, J. Critical Properties of Binary and Ternary Mixtures of Hexane + Methanol, Hexane + Carbon Dioxide, Methanol + Carbon Dioxide, and Hexane + Carbon Dioxide + Methanol. J. Chem. Eng. Data 2003, 48, 1610-1613.

[5] Demirbas, M. F.; Balat, M. Recent Advances on the Production and Utilization Trends of Bio-Fuels: a Global Perspective. Energy Conversion and Management 2006, 47, 2371-2381.

[6] Oliveira, M. B.; Queimada; A. J.; Coutinho, J. A. P. Prediction of Near and Supercritical Fatty Acid Ester + Alcohol Systems with the CPA EoS. J. Supercrit. Fluids 2010, 52, 241-248.

[7] Arcia, M. E.; Gerder, Y.; Gil, J. A Novel Improved Condensate-Recovery Method by Cyclic Supercritical CO2 Injection. SPE 2007, SPE107283-MS.

[8] Seyyedsar, M. S.; Farzaneh, A. S.; Sorahbi, M. Enhanced Heavy Oil Recovery by Intermittent $\mathrm{CO} 2$ Injection. SPE 2015, SPE-175140-MS.

[9] Kiselev, S. B. Cubic crossover equation of state. Fluid Phase Equilib. 1998, 147, 7-23.

[10] Kiselev, S. B.; Friend, D. G. Cubic Crossover Equation of State for Mixtures. Fluid Phase Equilib 1999, 162, 51-82.

[11] Anisimov, M. A.; Sengers, J. V. Critical and Crossover Phenomena in Fluids and Fluid Mixtures. Supercritical Fluids, 89-121, Springer: Netherlands, 2000.

[12] Sengers, J. V.; Sengers, J. M. H. L. Thermodynamic behavior of fluids near the critical point. Annu. Rev. Phys. Chem. 1986, 37, 189-222.

[13] Salvino, L. W.; White, J. A. Calculation of density fluctuation contributions to thermodynamic properties of simple fluids. J. Chem. Phys. 1992, 96, 4559-4568.

[14] Yan, W.; Varzandeh, F.; Stenby, E. H. PVT Modeling of Reservoir Fluids Using PC-SAFT EoS and Soave-BWR EoS. Fluid Phase Equilib. 2015, 386, 96-124. 
[15] Valderrama, J. O. The State of the Cubic Equations of State. Ind. Eng. Chem. Res. 2003, 42, 1603-1618.

[16] Soave, G. S. An Effective Modification of the Benedict-Webb-Rubin Equation of State. Fluid Phase Equilib. 1999, 164, 157-172.

[17] Kunz, O.; Klimeck, R.; Wagner, W.; Jaeschke, M. The GERG-2004 Wide-range Equation of State for Natural Gases and Other Mixtures. VDI Verlag GmbH: Düsseldorf, 2007.

[18] Kunz, O.; Wagner, W. The GERG-2008 wide-range equation of state for natural gases and other mixtures: an expansion of GERG-2004. J. Chem. Eng. Data, 2012, 57, 3032-3091.

[19] Kiselev, S. B.; Ely, J. F. Simplified crossover SAFT equation of state for pure fluids and fluid mixtures. Fluid Phase Equilib. 2000, 174, 93-113.

[20] White, J. A. Contribution of fluctuations to thermal properties of fluids with attractive forces of limited range: theory compared with $\mathrm{P} \rho \mathrm{T}$ and $\mathrm{C}_{\mathrm{v}}$ data for argon. Fluid Phase Equilib. 1992, 75, 53-64.

[21] Wilson, K. G. Renormalization Group and Critical Phenomena. I. Renormalization Group and the Kadanoff Scaling Picture. Physical Rev. B 1971, 4, 3174-3183.

[22] Chen, Z. Y.; Albright, P. C.; Sengers, J. V. Crossover from Singular Critical to Regular Classical Thermodynamic Behavior of Fluids. Phys. Rev. A 1990, 41, 3161-3177.

[23] Kiselev, S. B.; Kostyukova, I. G.; Povodyrev, A. A. Universal Crossover Behavior of Fluids and Fluid Mixtures in the Critical Region. Int. J. Thermophys. 1991, 12, 877-895.

[24] Jin, G. X.; Tang, S.; Sengers, J. V. Global Thermodynamic Behavior of Fluid Mixtures in the Critical Region. Fluid Phase Equilib. 1992, 75, 1-10.

[25] McCabe, C.; Kiselev, S. B. Application of Crossover Theory to the SAFT-VR Equation of State: SAFT-VRX for Pure Fluids. Ind. Eng. Chem. Res. 2004, 43, 2839-2851.

[26] Kiselev, S. B.; Ely, J. F.; Tan, S. P.; Adidharma, H.; Radosz, M. HRX-SAFT Equation of State for Fluid Mixtures: Application to Binary Mixtures of Carbon Dioxide, Water, and Methanol. Ind. Eng. Chem. Res. 2006, 45, 3981-3990.

[27] Janacek, J.; Paricaud, P.; Dicko, M. and Coquelet, C. A Generalized Kiselev Crossover Approach Applied to Soave-Redlich-Kwong Equation of State. Fluid Phase Equilib. 2015, 401, 16-26.

[28] White, J. A.; Zhang, S. Renormalization Group Theory for Fluids to Greater Density Distances from the Critical Point. Int. J. of Thermophys. 1998, 19, 1019-1027.

[29] Wilson, K. G.; Fisher, M. E. Critical Exponents in 3.99 Dimensions. Phys. Rev. Lett. 1972, 28, 240243.

[30] Cai, J.; Prausnitz, J. M. Thermodynamics for Fluid Mixtures near to and far from the Vapor-Liquid Critical Point. Fluid Phase Equilib. 2004, 219, 205 217.

[31] Cai, J.; Qiu, D.; Zhang, L.; Hu, Y. Vapor-Liquid Critical Properties of Multi-Component Fluid Mixture. Fluid Phase Equilib. 2006, 241, 229-235.

[32] Xu, X. H.; Duan, Y. Y. Crossover CPA Equation of State for Associating Fluids. Fluid Phase Equilib. 2010, 290, 148-152.

[33] Xu, X. H.; Duan, Y. Y. Prediction of the Critical Properties of Binary Alkanol + Alkane Mixtures Using a Crossover CPA Equation of State. Fluid Phase Equilib. 2011, 309, 168-173.

[34] Llovell, F.; Pamies, J. C.; Vega, L. F. Thermodynamic properties of Lennard-Jones chain molecules: Renormalization-group corrections to a modified statistical associating fluid theory. J. Chem. Phys. 2004, 121, 10715-10724.

[35] Llovell, F.; Vega, L. F. Global Fluid Phase Equilibria and Critical Phenomena of Selected Mixtures Using the Crossover Soft-SAFT Equation. J. Phys. Chem. B 2006, 110, 1350-1362.

[36] Llovell, F.; Vega, L. Phase Equilibria, Critical Behavior and Derivative Properties of Selected nAlkane/n-Alkane and n-Alkane/1-Alkanol Mixtures by the Crossover Soft-SAFT Equation of State. J. Supercrit. Fluids 2007, 41, 204-216.

[37] White, J. A.; Zhang, S. Renormalization group theory for fluids. J. Chem. Phys. 1993, 99, 20122019.

[38] White, J. A.; Zhang, S. Renormalization theory of nonuniversal thermal properties of fluids. J. Chem. Phys. 1995, 103, 1922-1928.

[39] Lue, L.; Prausnitz, J. M. Renormalization-group corrections to an approximate free-energy model for simple fluids near to and far from the critical region. J. Chem. Phys. 1998, 108, 5529-5536.

[40] Jiang, J.; Prausnitz, J. M. Critical temperatures and pressures for hydrocarbon mixtures from an equation of state with renormalization-group theory corrections. Fluid Phase Equilib. 2000, 169, 127147.

[41] White, J. A. Global renormalization calculations compared with simulations for Lenard-Jones fluid. J. 
Chem. Phys. 2000, 112, 3236-3243.

[42] Carnahan, N. F.; Starling, K. E. Equation of State for Nonattracting Rigid Spheres. J. Chem. Phys. 1969, 51, 635-636.

[43] Fisher, M. E. Renormalization of Critical Exponents by Hidden Variables. Physical Review 1968, 176, 257-272.

[44] Griffiths, R. B.; Wheeler, J. C. Critical Points in Multicomponent Systems. Phys. Rev. A 1970, 2, 1047-1064.

[45] Michelsen, M. L. Robust and Efficient Solution Procedures for Association Models. Ind. Eng. Chem. Res. 2006, 45, 8449-8453.

[46] Peneloux, A.; Rauzy, E.; Freze, R. A Consistent Correction for Redlich-Kwong-Soave Volumes. Fluid Phase Equilib. 1982, 8, 7-23.

[47] Pellegrini, L. A.; Moioli, S.; Gamba, S.; Ceragioli, P. Prediction of volumetric properties of fluids for oil and gas applications. Fluid Phase Equilib. 2012, 317, 52-58.

[48] Vinhal, A. P. C. M.; Yan, W.; Kontogeorgis, G. M. Evaluation of equations of state for simultaneous representation of phase equilibrium and critical phenomena. Fluid Phase Equilib. 2017, 437, 140154.

[49] Kontogeorgis, G. M.; Voutsas, E. C.; Yakoumis, I. V.; Tassios, D. P. An equation of state for associating fluids. Ind. Eng. Chem. Res. 1996, 43104318.

[50] Wertheim, M. S. Fluids with Highly Directional Attractive Forces. I. Statistical Thermodynamics. J. Stat. Phys. 1984, 35, 19-34.

[51] Kontogeorgis, G. M.; Folas, G. K. Thermodynamic Models for Industrial Applications. From Classical and Advanced Mixing Rules to Association Theories. Wiley \& Sons: New York, 2010.

[52] Yakoumis, I. V.; Kontogeorgis, G. M.; Voutsas, E. C.; Tassios, D. P. Vapor-Liquid Equilibria for Alcohol/Hydrocarbon Systems Using the CPA Equation of State. Fluid Phase Equilib. 1997, 130, 31-47.

[53] Polishuk, I.; Wisniak, J.; Segura, H. Prediction of the critical locus in binary mixtures using equation of state: I. Cubic equations of state, classical mixing rules, mixtures of methane-alkanes. Fluid Phase Equilib. 1999, 164, 13-17.

[54] van Konynenburg; P. H.; Scott, R. L. Critical lines and phase equilibria in binary van der Waals mixtures. Philos. Trans. R. Soc. London, Ser. A, 298, 495-540.
[55] Wichterle, I.; Kobayashi, R. Vapor-Liquid Equilibrium of Methane-Ethane System at Low Temperatures and High Pressures. Chem. Eng. Data 1972, 17, 9-12.

[56] Davalos, J.; Anderson, W. R.; Phelps, R. E.; Kidnay, A. J. Liquid-vapor equilibria at 250.00.deg.K for systems containing methane, ethane and carbon dioxide. Chem. Eng. Data 1976, 21, 81-84.

[57] Sage, B. H.; Lacey, W. N.; Schaafsma, J. G.; Phase Equilibria in Hydrocarbon Systems II. MethanePropane System. Ind. Eng. Chem. 1934, 26, 214217.

[58] Sage, B. H.; Hicks, B. L.; Lacey, W. N. Phase Equilibria in Hydrocarbon Systems: The Methane-nButane System in the Two-Phase Region. Ind. Eng. Chem. 1940, 32, 1085-1092.

[59] Roberts, L. R.; Wang, R. H.; Azarnoosh, A.; McKetta, J. J. Methane-n-Butane System in the Two-Phase Region. Chem. Eng. Data 1962, 7, 484485.

[60] Berry, V. M.; Sage, B. H. Phase Behavior in Binary and Multicomponent Systems At Elevated Pressures: n-Pentane and Methane-n -Pentane. National Bureau of Standards, Pasadena, California, US, 1970.

[61] Poston, R. S.; McKetta, J. J. Vapor-Liquid Equilibrium in the Methane-n-Hexane System. Chem. Eng. Data 1966, 11, 362-363.

[62] Reamer, H. H.; Sage, B. H.; Lacey, W. N. Phase Equilibria in Hydrocarbon Systems. Volumetric and Phase Behavior of the Methane-n-Heptane System. Ind. Chem. Eng. Data Series 1956, 1, 29-42.

[63] Shipman, L. M.; Kohn, J. P. Heterogeneous Phase and Volumetric Equilibrium in the Methane-nNonane System. J. Chem. Eng. Data 1966, 11, 176180.

[64] Reamer, H. H.; Olds, R. H.; Sage, B. H.; Lacey, W. N. Phase Equilibria in Hydrocarbon Systems: Methane-Decane System. Ind. Eng. Chem. 1942, 34, 1526-1531.

[65] Hicks, C. P.; Young, C. L. The Gas-Liquid Critical Properties of Binary Mixtures. Chem. Rev. 1975, 75, 119-175.

[66] Cheng, H.; Anisimov, M. A.; Sengers, J. V. Prediction of thermodynamic and transport properties in the one-phase region of methane $+\mathrm{n}$ hexane mixtures near their critical end points. Fluid Phase Equilib. 1997, 128, 67-96. 


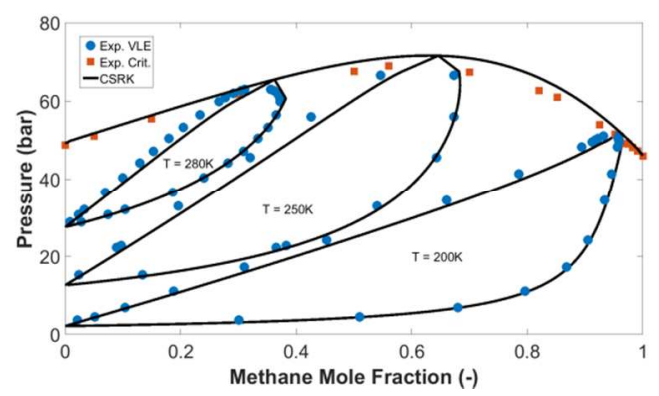

For Table of Contents only 